OPEN ACCESS

Edited by:

Jill Kolesar,

University of Kentucky, United States

Reviewed by:

James William Jacobberger, Case Western Reserve University,

United States

Alice Mims,

The Ohio State University,

United States

Steven Gore,

National Cancer Institute (NCl),

United States

*Correspondence:

Curt I. Civin

Ccivin@som.umaryland.edu

Michelle A. Rudek

mrudek2@jhmi.edu

${ }^{\dagger}$ Present address:

Blake S. Moses,

Jacobio Pharmaceuticals Inc.,

Lexington, MA, United States

${ }^{\mp}$ These authors have contributed

equally to this work and

share first authorship

$\S$ These authors have contributed equally to this work and share senior authorship

Specialty section:

This article was submitted to Pharmacology of Anti-Cancer Drugs, a section of the journal Frontiers in Oncology

Received: 05 October 2021 Accepted: 17 December 2021

Published: 11 January 2022

Citation:

Kagan AB, Moses BS, Mott BT, Rai G, Anders NM, Rudek MA and Civin Cl (2022) A Novel 2-Carbon-

Linked Dimeric Artemisinin With

Potent Antileukemic Activity and

Favorable Pharmacology.

Front. Oncol. 11:790037.

doi: 10.3389/fonc.2021.790037

\section{A Novel 2-Carbon-Linked Dimeric Artemisinin With Potent Antileukemic Activity and Favorable Pharmacology}

\author{
Amanda B. Kagan ${ }^{1 \neq}$, Blake S. Moses ${ }^{2 t \neq}$, Bryan T. Mott ${ }^{3 \neq}$, Ganesha Rai ${ }^{4}$, \\ Nicole M. Anders ${ }^{5}$, Michelle A. Rudek ${ }^{1,5 \star \S}$ and Curt I. Civin ${ }^{2 \star \S}$ \\ 1 Department of Medicine, Division of Clinical Pharmacology, School of Medicine, Johns Hopkins University, Baltimore, \\ MD, United States, ${ }^{2}$ Center for Stem Cell Biology \& Regenerative Medicine, Marlene and Stewart Greenebaum Comprehensive \\ Cancer Center, Departments of Pediatrics and Physiology, School of Medicine, University of Maryland, Baltimore, MD, United \\ States, ${ }^{3}$ Department of Neurosurgery, Wake Forest Baptist Health, Winston-Salem, NC, United States, ${ }^{4}$ Department of Pre- \\ Clinical Innovation, National Center for Advancing Translational Sciences, National Institutes of Health, Bethesda, MD, United \\ States, ${ }^{5}$ Department of Oncology, School of Medicine, Johns Hopkins University, Baltimore, MD, United States
}

Acute myeloid leukemia (AML) remains a devastating disease, with low cure rates despite intensive standard chemotherapy regimens. In the past decade, targeted antileukemic drugs have emerged from research efforts. Nevertheless, targeted therapies are often effective for only a subset of patients whose leukemias harbor a distinct mutational or gene expression profile and provide only transient antileukemic responses as monotherapies. We previously presented single agent and combination preclinical data for a novel 3carbon-linked artemisinin-derived dimer (3C-ART), diphenylphosphate analog 838 (ART838), that indicates a promising approach to treat AML, given its demonstrated synergy with targeted antileukemic drugs and large therapeutic window. We now report new data from our initial evaluation of a structurally distinct class of 2-carbon-linked dimeric artemisinin-derived analogs (2C-ARTs) with prior documented in vivo antimalarial activity. These 2C-ARTs have antileukemic activity at low (nM) concentrations, have similar cooperativity with other antineoplastic drugs and comparable physicochemical properties to ART838, and provide a viable path to clinical development.

Keywords: artemisinins, sorafenib, venetoclax (ABT-199), leukemia, antineoplastics

\section{INTRODUCTION}

The prognosis for patients with acute myeloid leukemia (AML) remains poor (5-year survival $\sim 25$ $30 \%$ ) and is particularly dismal for patients who are over 60 years old, unfit, or with relapsed/ refractory disease, unfavorable cytogenetics, or certain molecular abnormalities (1). According to the Surveillance, Epidemiology, and End Results (SEER) database from 2010-2017, median overall survival in de novo AML was only 11 months (2). Improved understanding of the pathophysiology and mutational landscape of AML has stimulated the successful development of a number of targeted therapies. In fact, nine new AML therapeutics - including inhibitors of B-cell lymphoma 2 (BCL2), FMS-like tyrosine kinase 3 (FLT3), and isocitrate dehydrogenase (IDH) - were approved by the FDA between 2017 and $2021(1,3)$. However, the use of these targeted inhibitors as monotherapies has achieved only shallow, transient responses followed by the emergence of 
therapy resistant AML in the majority of patients with targetable mutations (4-6). There has thus been growing interest in additional new agents and combination regimens with novel mechanisms that may be non-cross resistant with respect to current antileukemic drugs, and that suggest low clinical toxicity.

The revolutionary antimalarial natural product artemisinin and its semi-synthetic derivatives (collectively referred to here as ARTs) (Figure 1) all share a unique endoperoxide pharmacophore that is bio-activated to cytotoxic carbon free radical species (7). Although they are best known as antimalarials, ARTs are active against a range of microbes (8-10) and also have potent antineoplastic activity (11-14). They are active against a broad array of leukemia cell lines, including those harboring poor risk genetic aberrations, such as FLT3 mutations and BCR-ABL and mixed-lineage leukemia (MLL) rearrangements, and those resistant to current antileukemic drugs (15-18). First-generation ART derivatives suffer from poor bioavailability and rapid metabolism (7), precluding the sustained exposure desired for antineoplastic therapy. Semi-synthetic trioxane dimers were designed to address these pharmacokinetic vulnerabilities (19). We previously reported that the novel 3-carbon linked ART dimer (3C-ART), diphenylphosphate analog 838 (ART838), was 88-fold more potent than artesunate (AS), the major clinical artemisinin derivative, against 23 leukemia cell lines, with a favorable therapeutic window (17). ART838 synergized with the kinase inhibitor sorafenib (SOR) and the BCL2 inhibitor venetoclax (VEN) (to form a combination regimen referred to as "SAV") both in vitro and in AML xenograft and PDX models (20). Unfortunately, commercial development of ART838 is impractical, as its patent protection ends soon (US7417156B2 issued 9/27/02).

A structurally distinct class of two-carbon linked ART dimers (2C-ARTs) was originally developed to further enhance druglike properties (19). We evaluated two of over 20 analogs from this 2C-ART class that were shown to be effective against an in vivo mouse model of malaria (19). Ketone analog ART576 and allyloxime analog ART631 were selected for evaluation due to their antimalarial efficacy and ease of synthesis. In addition, for both these ART analogs, there was unpublished in vitro data demonstrating potent activity against a range of cancer cell lines, including several leukemia cell lines. ART576 and ART631 (Figure 1) were evaluated for in vitro and in vivo antileukemic efficacy, both as monotherapies and in combination with SOR and VEN, as well as in pharmacodynamic and pharmacokinetic (PK) assays.

\section{MATERIALS AND METHODS}

\section{Reagents}

Synthesis of 2C-ARTs has been described previously (19). All other compounds, antibodies, and reagents were as detailed in Supplemental Table 1 of Moses et al. (20).

\section{Cells}

The AML cell lines used for in vitro antileukemic activity assays are described in Table 1. Lentiviral-transduced luciferase (luc)labeled MV4;11 and MOLM14 AML cells were developed and characterized previously $(20,21)$.

\section{Cytotoxicity}

Cytotoxicity was assessed using alamarBlue assays (Life Technologies, Grand Island, NY) following manufacturer's guidelines. Cell death was verified by Annexin V/7aminoactinomycin D (7AAD) staining (BioLegend, San Diego, CA) per manufacturer's guidelines.

\section{Metabolic Stability}

The metabolic stability of ART631 was assessed in human and mouse plasma and liver microsomes (LM). For phase I microsomal stability assays, analogs ( $10 \mu \mathrm{M}$ in $<1 \%$ acetonitrile) were incubated in PBS containing liver microsomes, with or without<smiles>COC1O[C@@H]2O[C@@]3(C)CCC4[C@@H](C)CCC(C1C)C42OO3</smiles><smiles>CC1OC2O[C@@]3(C)CCC4OO[C@@]23C1CC[C@H]4C</smiles>

C

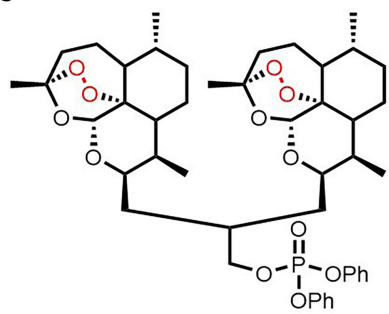

D

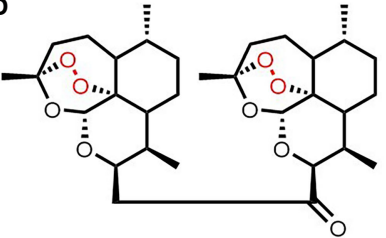

E

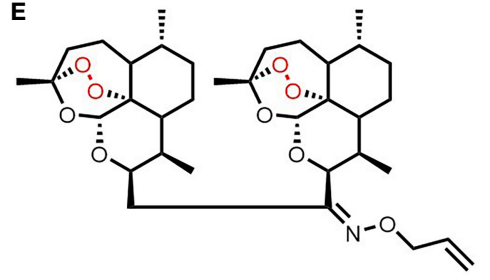

FIGURE 1 | Chemical structures of artemisinin (A), artesunate (B), ART838 (C), ART576 (D) and ART631 (E) 
TABLE 1 | AML cell lines.

\begin{tabular}{|c|c|c|c|c|}
\hline Cell Line & Major Genetic Aberrations & Source & Catalog \# & Culture Media \\
\hline K562 & BCR-ABL, TP53 & ATCC, Manassas, VA & CCL-243 & $\mathrm{RPMI}+10 \% \mathrm{FBS}$ \\
\hline KG1a & FGFR1OP2-FGFR1, TP53 & ATCC, Manassas, VA & CCL-240 & RPMI+10\%FBS \\
\hline HEL & JAK2V617F & ATCC, Manassas, VA & TIB-180 & RPMI+10\%FBS \\
\hline HL60 & PML-RARA, c-MYC & ATCC, Manassas, VA & CCL-246 & RPMI+10\%FBS \\
\hline ML2 & KMT2A-MLLT4, KRAS, TP53 & DSMZ, Branschweig, DEU & ACC-15 & RPMI+10\%FBS \\
\hline MOLM14 & KMT2A-MLLT3, FLT3ITD, CBL $\Delta$ exon 8 & DSMZ, Branschweig, DEU & ACC-777 & RPMI+10\%FBS \\
\hline MV4;11 & KMT2A-AFF1, FLT3ITD & ATCC, Manassas, VA & CRL-9591 & $\mathrm{RPMI}+10 \% \mathrm{FBS}$ \\
\hline TF1 & EpoR truncation, IDH2 & ATCC, Manassas, VA & CCL-2003 & RPMI+10\% FBS+0.5uL/mL GMCSF \\
\hline THP1 & KMT2A-MLLT3, NRAS, TP53 & ATCC, Manassas, VA & TIB-202 & RPMI+10\% FBS+50uM 2-Mercaptoethanol \\
\hline U937 & PICALM-AF10, TP53 & ATCC, Manassas, VA & CRL-1593 & RPMI+10\%FBS \\
\hline
\end{tabular}

These AML cell lines utilized for in vitro antileukemic activity testing $\left(I C_{50}\right)$ were purchased from the listed sources and cultured in the indicated media. Their major genetic aberrations, including oncogenic gene fusions, FLT3ITD status, and point mutations, are as listed.

cofactors (22). Microsomal controls (without cofactors) were used to determine non-enzymatic degradation. All experiments were incubated at $37^{\circ} \mathrm{C}$ for $1 \mathrm{~h}$ and performed in duplicate. Reactions were stopped with acetonitrile and analyzed as described below.

\section{Reactive Oxygen Species (ROS)}

Cells were pre-treated with $5 \mathrm{mM}$ ROS detection reagent 5-(and 6-)-chloromethyl-2',7'-dichlorodihydrofluorescein diacetate (CM-H2DCFDA, Life Technologies) for $30 \mathrm{~min}$, followed by the iron chelator deferoxamine mesylate (DFO; $11 \mathrm{mM})$ for 60 min. Cells were then treated with ART631 (200 nM) or DMSO control for $18 \mathrm{~h}$, with subsequent analysis by flow cytometry. Western blotting and quantitative reverse transcription PCR (qRT-PCR) to assess for molecular markers of ROS-mediated apoptosis were performed as previously described (20).

\section{Pharmacokinetics (PK)}

Mice were dosed orally with ART631 at empirically determined maximally tolerated doses (MTDs) of $75 \mathrm{mg} / \mathrm{kg}$ ART631 as a single dose alone or $15 \mathrm{mg} / \mathrm{kg}$ as a multiple dose regimen (once daily x5), either alone or in combination with SOR $(30 \mathrm{mg} / \mathrm{kg} / \mathrm{d})$ and VEN $(150 \mathrm{mg} / \mathrm{kg} / \mathrm{d})$, for side-by-side comparison to the ART838, SOR and VEN combination evaluated previously (20). ART631, SOR, and VEN were mixed in vehicle and given as a single administration. Mice ( $\mathrm{n}=3$ /time point) were euthanized at $0.5,1,1.5,2,3,4,6,8$ and $12 \mathrm{~h}$ after dosing. ART631 was quantified in plasma as described below. Pharmacokinetic parameters were calculated from mean concentration-time data using non-compartmental methods in Phoenix WinNonlin version 8.3 (Certara, Princeton, NJ). The maximum plasma concentration $\left(\mathrm{C}_{\max }\right)$ and time to $\mathrm{C}_{\max }\left(\mathrm{T}_{\max }\right)$ were the observed values. The $\mathrm{AUC}_{\text {last }}$ was calculated using the loglinear trapezoidal method. AUC was extrapolated to infinity (AUC $\mathrm{INF}_{\mathrm{INF}}$ ) by dividing the last quantifiable concentration by the terminal disposition rate constant $\left(\lambda_{\mathrm{z}}\right)$. The $\lambda_{\mathrm{z}}$ was determined from at least 3 points on the slope of the terminal phase of the concentration-time profile. The terminal half-life $\left(\mathrm{T}_{1 / 2}\right)$ was determined by dividing 0.693 by $\lambda_{\mathrm{z}}$. Apparent clearance $(\mathrm{Cl} / \mathrm{F})$ was calculated by dividing the dose administered by $\mathrm{AUC}_{\mathrm{INF}}$. Apparent volume of distribution $(\mathrm{V} / \mathrm{F})$ was calculated by dividing $\mathrm{Cl} / \mathrm{F}$ by $\lambda_{z}$. If the percent AUC extrapolated was $>20 \%$ or the $\mathrm{r}^{2}$ of $\lambda_{\mathrm{z}}$ was $<0.85$, the $\mathrm{AUC}_{\mathrm{INF}}, \mathrm{Cl} / \mathrm{F}, \mathrm{T}_{1 / 2}$ and $\mathrm{V} / \mathrm{F}$ were not reported.

The Method of Bailer was used to estimate the variance of AUC given the calculated variance of the mean concentration at each time point (23). A pairwise comparison utilizing $\mathrm{Z}$ test was used to determine whether there was a significant difference between ART631 exposures as expressed by $\mathrm{AUC}_{\text {last }}$ (24). In all cases, $\mathrm{p}<0.05$ was considered statistically significant.

\section{Bioanalytical Method}

ART631 was extracted from $25 \mu \mathrm{L}$ plasma or microsomal solutions with $100 \mu \mathrm{L}$ acetonitrile containing artemisinin (i.e. the natural product) as internal standard. Chromatographic separation was achieved with a Zorbax XDB C18 column (2.1 x 50mm, $3.5 \mu \mathrm{m}$, Agilent Technologies, Santa Clara, CA) at room temperature using gradient elution over a 6 min total analytical run time. Mobile phase A was ammonium acetate $(2 \mathrm{mM})$ containing $0.1 \%$ formic acid, and mobile phase B was acetonitrile containing $0.1 \%$ formic acid. The gradient started with mobile phase B and was held at $40 \%$ for 0.5 minutes with a flow rate of $0.4 \mathrm{~mL} / \mathrm{min}$, then increased to $100 \%$ over 1.5 minutes and held for 2.0 minutes, and finally returned back to $40 \%$ mobile phase $\mathrm{B}$ and allowed to equilibrate for 2.0 minutes. An $\mathrm{AB}$ Sciex 5500 triple quadrupole mass spectrometer operated in positive electrospray ionization mode was used for the detection of ART631. For stability studies, the ratio of ART631 to the internal standard, artemisinin, was assessed. Calibration curves for ART631 were computed using the ratio of the peak area to that of the internal standard by using a quadratic equation with a $1 / \mathrm{x}^{2}$ weighting function over the range of 8 $1585 \mathrm{nM}$ with dilutions of up to $1: 10(\mathrm{v} / \mathrm{v})$.

\section{AML (Cell Line) Xenograft and Patient- Derived Xenograft (PDX) Assays}

Immunodeficient NOD-Rag1 ${ }^{\text {null } I L 2} 2 g^{\text {null }}$ (NRG) mice were purchased from Jackson Laboratory (Bar Harbor, ME), bred and housed at the University of Maryland Baltimore. Similar to our previous publication (20). mice were transplanted IV with luc-labeled human AML cell lines or cryopreserved AML patient cells on day -10 , and baseline total body luminescence was quantified on day 0 by Xenogen bioluminescence imaging (Xenogen IVIS Spectrum; PerkinElmer, Waltham, MA). Mice 
were allocated to treatment groups so that each group had similar average day 0 luminescence, then groups were administered drugs per oral (PO; gavage). Luminescence of each mouse was assessed over time and compared with that mouse's day 0 luminescence (fold-change AML burden). Clinical behavior, appearance, weight, and survival were also monitored.

\section{RESULTS}

\section{ART576 and ART631 Exhibited In Vitro Activity Against Multiple AML Cell Lines at Nanomolar Concentrations}

In alamarBlue assays, ART838, ART631, and ART576 had comparable in vitro activity against the human MOLM14 AML cell line, and all 3 were substantially more potent than the firstgeneration derivative artesunate (AS) (Figure 2). ART576 inhibited growth of 8 of 10 human AML cell lines tested, and ART576's antileukemic potency ( $\mathrm{IC}_{50}$ range: 23-105 nM; Table 2) was similar to that of ART838, which inhibited 9 of the same 10 cell lines ( $\mathrm{IC}_{50}$ range: 22-112 nM). ART576 did not inhibit THP1 cell growth ( $\mathrm{IC}_{50}>1000 \mathrm{nM}$ ), while ART838 did $\left(\mathrm{IC}_{50}=45 \mathrm{nM}\right)$. ART631 inhibited growth of the same 9 of these 10 human AML cell lines ( $\mathrm{IC}_{50}$ range: $20-45 \mathrm{nM}$; Table 2 ) as did ART838, slightly exceeding the potency of ART838. Cell lines with adverse risk cytogenetic and molecular features, e.g. MLL rearrangements (ML2, MOLM14, MV4;11, THP1), FLT3-ITD (MOLM14, MV4;11), TP53 mutations (KG1a, ML2, THP1, U937), and NRAS/KRAS mutations (ML2, THP1), were among those exquisitely sensitive to ART631, with $\mathrm{IC}_{50} \mathrm{~s}<50$ nM. The K562 cell line was the only exception, as it was resistant to all 3 of these ARTs. Based on this in vitro activity data combined with the drug stability data described below, ART631 was prioritized over ART576 for further in vitro and in vivo antileukemic efficacy experiments here (Table 2).

\section{ART631 Was More Stable Than ART576 in Microsomes}

We compared the in vitro stability of ART576 and ART631 (Table 3). ART576 was stable in plasma ( $<10 \%$ degradation), but unstable with degradation of $>70 \%$ in human liver microsomes with co-factors and $>90 \%$ in mouse liver microsomes with cofactors (Table 3). Compared to ART576, ART631 was less stable in plasma with degradation of $>40 \%$, but was considerably less susceptible to NADPH-dependent phase I metabolism, with $\sim 30 \%$ degradation in human liver microsomes with co-factors and $\sim 40 \%$ in mouse liver microsomes with co-factors (Table 3 ). Based on these results as well as the in vitro antileukemic activity results above, we prioritized ART631 over ART576 for in vivo PK profiling and antileukemic efficacy assays, despite the fact that neither were equivalent to ART838 in stability $[\leq 20 \%$ degradation as previously reported (20)].

\section{ART631 Synergized With the Kinase Inhibitor Sorafenib (SOR) and the BCL2 Inhibitor Venetoclax (VEN) to Cause Apoptotic AML Cell Death}

MOLM14, MV4;11, and HL60 human AML cells were assessed for cell viability and caspase-dependent apoptosis following treatment with ART631 (200 nM), VEN (50 nM), SOR (5 $\mu \mathrm{M})$, and combinations thereof. These concentrations were chosen because they resulted in less than $15 \%$ cell death in these cell lines when drugs were given as single agents (20).

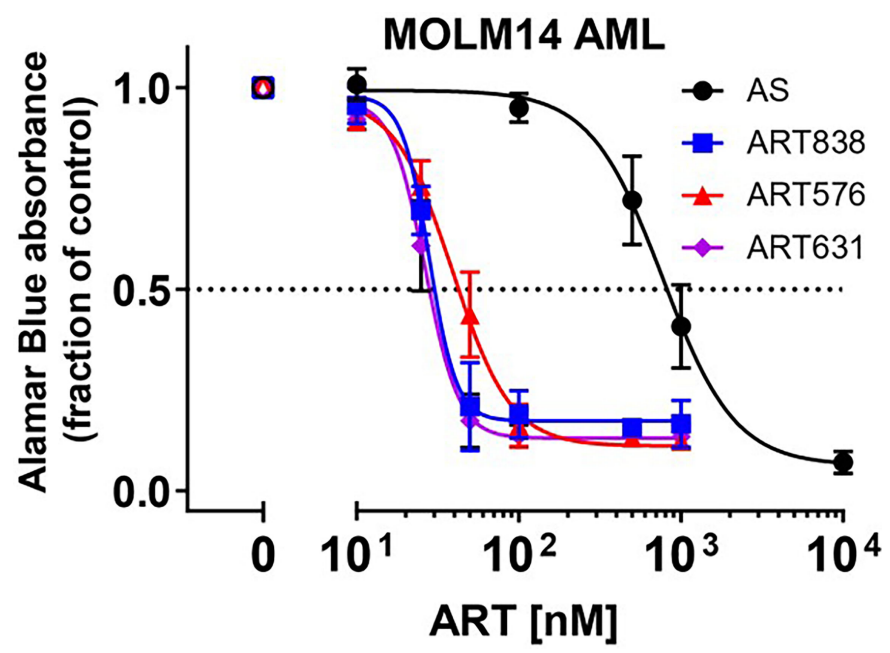

FIGURE 2 | 2C-ARTs ART631 and ART576 potently inhibited the growth of MOLM14 AML cells, with activity comparable to ART838. In this experiment, provided to show the entire concentration:response range, MOLM14 AML cells were cultured with a range of concentrations of artesunate (AS), ART838, ART631, and ART576 for 48h. Graph indicates growth inhibition relative to vehicle (DMSO)-treated controls by alamarBlue absorbance assays. Data points and error bars represent mean and SD of 3 independent experiments done in triplicate. Although Table 1 shows only $\mathrm{IC}_{50} \mathrm{~S}$, this full concentration:response range was assessed for all $10 \mathrm{AML}$ cell lines. 
TABLE 2 | In vitro $I_{50}$ values for ART analogs against 10 human AML cell lines.

\begin{tabular}{|c|c|c|c|c|}
\hline \multirow[t]{2}{*}{ AML } & \multicolumn{4}{|c|}{$\mathrm{IC}_{50}(\mathrm{nM})$} \\
\hline & ART631 & ART576 & ART838 & AS \\
\hline K562 & $>1000$ & $>1000$ & $>1000$ & $>10000$ \\
\hline KG1a & $29 \pm 2$ & $64 \pm 4$ & $112 \pm 7$ & $2603 \pm 458$ \\
\hline HEL & $45 \pm 5$ & $80 \pm 19$ & $72 \pm 6$ & $1114 \pm 93$ \\
\hline HL60 & $41 \pm 9$ & $72 \pm 18$ & $45 \pm 3$ & $1057 \pm 83$ \\
\hline ML2 & $34 \pm 4$ & $105 \pm 12$ & $40 \pm 5$ & $1578 \pm 94$ \\
\hline MOLM14 & $32 \pm 4$ & $43 \pm 7$ & $28 \pm 3$ & $890 \pm 99$ \\
\hline MV4;11 & $27 \pm 2$ & $38 \pm 10$ & $28 \pm 1$ & $610 \pm 30$ \\
\hline TF1 & $27 \pm 2$ & $49 \pm 12$ & $36 \pm 3$ & $1715 \pm 302$ \\
\hline THP1 & $42 \pm 2$ & $>1000$ & $45 \pm 3$ & $>10000$ \\
\hline U937 & $20 \pm 5$ & $23 \pm 1$ & $22 \pm 3$ & $521 \pm 96$ \\
\hline
\end{tabular}

AML cell lines were cultured with a range of concentrations of $A S$, ART838, ART631, or ART576 for 48h. IC 50 s were calculated based on observed growth inhibition relative to vehicle (DMSO)-treated controls using alamarBlue absorbance assays (means of 2 independent alamarBlue assays performed with triplicate samples \pm SD).

Consistent with published data for ART838 (20), there was substantially more synergy between ART631 and VEN than between ART631 and SOR in all three cell lines, and the VEN plus SOR combination was also highly effective (Figure 3). Importantly, treatment with ART631 plus VEN plus SOR as a 3 -drug combination induced apoptotic cell death more potently than any of the 2-drug combinations, although there was only modest superiority of the ART631 plus VEN plus SOR 3-drug combination over the ART631 plus VEN or the VEN plus SOR 2-drug combinations (Figure 3). Pre-treatment with the pancaspase inhibitor QVD-OPh (QVD) almost fully inhibited apoptosis and cell death for all of these drugs and combinations.

\section{ART631 Elevated ROS and DDIT3/CHOP Levels, and Reduced MCL1 Protein Levels in AML Cells}

The accepted antimicrobial and antineoplastic mechanism of ARTs involves bioactivation of the endoperoxide pharmacophore by $\mathrm{Fe}^{2+}$-containing molecules, resulting in generation of reactive oxygen species (ROS) and free radicals (25). Consistent with this mechanism, treatment of MOLM14 cells with ART631 (200 nM) substantially increased total cellular ROS levels (Figure 4A), and pre-treatment with the iron chelator deferoxamine mesylate (DFO; $11 \mathrm{uM}$ ) diminished ROS generation by ART631, as demonstrated previously for ART838 (17). There was an associated increase in the level of C/EBP homologous protein $(\mathrm{CHOP})$, a protein well known to play an important role in ROS-induced apoptosis (18), following treatment with ART631 (Figure 4B), comparable to that observed with ART838. Consistent with CHOP protein elevation, DDIT3, the mRNA which encodes CHOP, was increased in
MOLM14 cells treated with ART631, ART838, or AS, by qRTPCR (Figure 4C), suggesting DDIT3 as an attractive potential pharmacodynamic biomarker. Last, like ART838 and other ARTs $(18,20)$, ART631 decreased anti-apoptotic protein MCL1 expression substantially, by western blotting of MOLM14 cells (Figure 4B). Western blotting to assess CHOP and MCL1 expression and qRT-PCR to assess DDIT3 mRNA levels were also performed in MV4;11 and ML2 AML cells with similar results (Figures 5A, B).

\section{Maximum Tolerated Dose (MTD) and PK of ART631 in NRG Mice}

In pilot experiments, the MTD of ART631 was determined, in NRG (host strain for our AML xenograft models) mice, to be 75 $\mathrm{mg} / \mathrm{kg}$ PO (gavage) for a single dose, or $15 \mathrm{mg} / \mathrm{kg} / \mathrm{d}$ PO x $5 \mathrm{~d}$ every 14 days. When ART631 was combined with $150 \mathrm{mg} / \mathrm{kg} / \mathrm{d}$ VEN and/or $30 \mathrm{mg} / \mathrm{kg} / \mathrm{d}$ SOR, as was done previously with ART838 (20), ART631 was tolerated at the multiple dose MTD, and no further dose adjustments were made. The PK of ART631 was assessed at the MTD.

Plasma concentration-time profiles following ART631 administration at the single and multiple dose MTD and in the SAV combination are presented in Figure 6, and associated PK parameters in Table 4. After PO administration, $\mathrm{C}_{\max }$ occurred at $1.5-2.0 \mathrm{~h}$ with a mono-exponential decline. The concentration of ART631 exceeded the in vitro $\mathrm{IC}_{50}$ for 9 of the $10 \mathrm{AML}$ cell lines tested $(45 \mathrm{nM})$ for at least $6 \mathrm{~h}$ after administration as a single dose, as multiple doses alone, or as multiple doses in the SAV combination (Figure 6). ART631's half-life of $1.1-1.7 \mathrm{~h}$ (alone) $2.4 \mathrm{~h}$ (in SAV) indicates that daily dosing is reasonable. Using the

TABLE 3 | Stabilities of ARTs.

\begin{tabular}{lccccc}
\hline \multirow{2}{*}{$\begin{array}{l}\text { Matrix } \\
\text { Cofactors }\end{array}$} & \multicolumn{2}{c}{ Plasma (EDTA) } & \multicolumn{3}{c}{ Phase I Metabolism } \\
\cline { 2 - 5 } & Mouse & Human & & MLM & HLM \\
\cline { 5 - 6 } & & & NADPH(-) & NADPH(+) & NADPH(-) \\
\hline ART576 & 101 & 91 & 96 & 7 & 89 \\
ART631 & 62 & 60 & 60 & 35 & 86 \\
\hline
\end{tabular}

2C-ART analogs were compared for stability in human and mouse plasma or in LM with or without cofactors. All values represent percentages remaining of the original ART analog. 


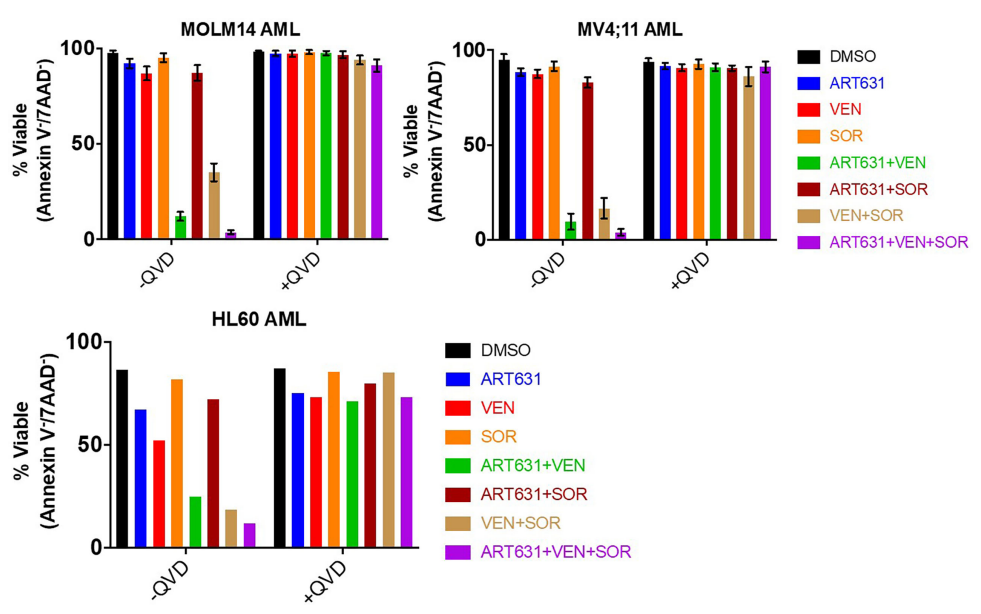

FIGURE 3 | ART631-based combination treatments reduced cell viability in AML cell lines. MOLM14, MV4;11, and HL60 AML cells were each treated for 18h with vehicle (DMSO) control, ART631 alone (200 nM), VEN alone (50 nM), SOR alone (5 uM), and 2- and 3-drug combinations, all with and without a $30-45$ min preincubation with $10 \mathrm{nM}$ pan-caspase inhibitor QVD-OPh (QVD). Treated cells were stained with $2.5 \mathrm{ug} / \mathrm{mL}$ 7-aminoactinomycin D (7-AAD) and 0.5 ug/mL allophycocyanin (APC)-conjugated Annexin V in Annexin V binding buffer and analyzed by flow cytometry. Graphs indicate \% cell viability based on Annexin V/7-AAD dual staining. For MOLM14 and MV4;11, means and SDs shown are based on 3 independent experiments. HL60 cell data is from one experiment done in triplicate.

Method of Bailer, the $\mathrm{AUC}_{\text {last }}$ from single dose ART631 (22905 $\mathrm{nM}^{\star} \mathrm{h}$ ) was significantly higher than $\mathrm{AUC}_{\text {last }}$ from multiple doses of ART631, either alone $\left(2737 \mathrm{nM}^{\star} \mathrm{h}\right)$ or when administered as part of the SAV combination $\left(3734 \mathrm{nM}^{\star} \mathrm{h}\right)$ (Table 4). The AUC last from multiple dose ART631 when administered in SAV $\left(3734 \mathrm{nM}^{\star} \mathrm{h}\right)$ was significantly higher compared to that of the multiple dose ART631 administered alone (2737 $\left.\mathrm{nM}^{\star} \mathrm{h}\right)$ (Table 4), but this increased exposure did not result in increased clinical toxicity.

\section{ART631-Containing SAV Induced Deep, Long Remissions in Two Human AML Cell Line Xenograft Models}

Using our previously determined 5-day MTD SAV drug schedule (i.e. 5-day treatment cycles consisting of $15 \mathrm{mg} / \mathrm{kg} / \mathrm{d}$ ART631 combined with $150 \mathrm{mg} / \mathrm{kg} / \mathrm{d}$ VEN and $30 \mathrm{mg} / \mathrm{kg} / \mathrm{d}$ SOR, followed by 9 days off drug; schematic in Figure 7A), we assessed treatment of established human luc-labeled MV4;11 (Figures 7B-D) and MOLM14 AML (Figures 8A-C) cell line xenografts with VEN plus SOR plus ART631 in NRG mice (Figures 7B, 8A). These xenograft experiments with ART631 and ART631-containing SAV were performed simultaneously with those with ART838 and ART838-containing SAV reported previously (20). Outcome metrics included in vivo antileukemic efficacy after completion of each treatment cycle relative to treatment day 0 , measured as foldchange in AML burden via Xenogen imaging (Figures 7C, 8B), and survival (Figures 7D, 8C).

Although no activity of ART631 monotherapy was observed in these experiments, ART631 was clinically tolerable, and the ART631-containing SAV regimen had potent antileukemic efficacy, comparable to that seen with the ART838-containing SAV regimen (20). In the MV4;11 xenograft model, 9 of 10 mice from the ART631-containing SAV group had undetectable AML burdens on day 98 (Figure 7B), compared with 5 of 9 mice from the ART838-containing SAV group (20); however, the increase in survival in the ART631-containing SAV group compared with the ART838-containing SAV group did not reach statistical significance $(\mathrm{p}=0.112)$. In the MOLM14 xenograft model, 2 of 10 mice from the ART631-containing SAV group had undetectable AML burdens on day 147 (Figure 8A), compared with 2 of 9 mice from the ART838-containing SAV group (20). Treatment ended on day 60 for both MV4;11 and MOLM14 xenograft models.

\section{ART631-Containing SAV Slowed Growth of an AML PDX Model}

We then compared the efficacy of $15 \mathrm{mg} / \mathrm{kg} / \mathrm{d}$ ART631 monotherapy, versus $150 \mathrm{mg} / \mathrm{kg} / \mathrm{d}$ VEN plus $30 \mathrm{mg} / \mathrm{kg} / \mathrm{d} \mathrm{SOR}$, versus ART631 combined with VEN plus SOR (i.e. SAV) at the previously indicated doses, using a luc-labeled AML45 PDX model (Figure 9A). This PDX experiment was performed simultaneously with the previously reported AML45 PDX experiment evaluating ART838 monotherapy and ART838containing SAV (20). All combination regimens [SOR plus VEN, ART631-containing SAV, and ART838-containing SAV (20)] slowed the increase in this patient-derived AML burden over time versus vehicle (Figure 9B). ART631 monotherapy resulted in a statistically significant increase in survival versus vehicle ( $\mathrm{p}=0.0029$; Figure 9C), though the magnitude was small; whereas the increase in survival did not reach statistical significance with ART838 monotherapy versus vehicle (20). Both SAV groups had longer survival than SOR plus VEN ( $\mathrm{p}=0.0002$ for ART838-containing SAV (20) and $\mathrm{p}<0.0001$ for ART631-containing SAV; Figure 9C). The ART631-containing SAV group had longer survival than the ART838-containing SAV group $(\mathrm{p}=0.0187)$ and a nearly twofold survival increment over vehicle (Figure 9C). 
A

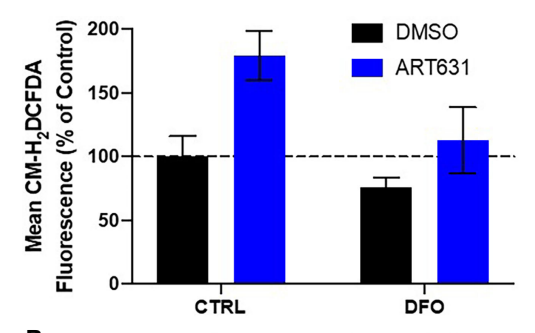

B

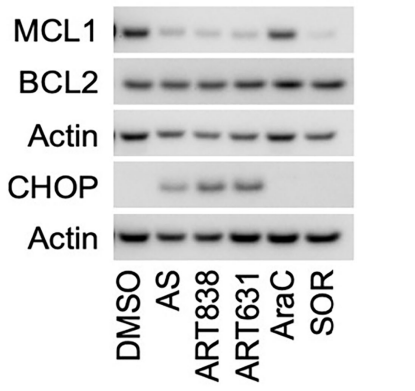

C

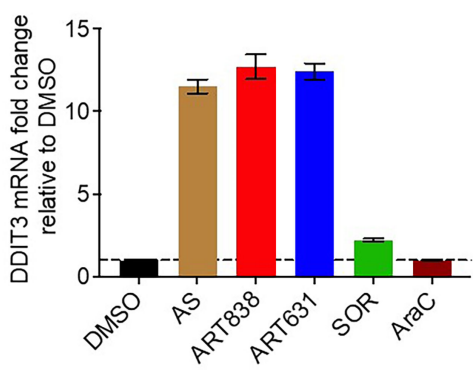

FIGURE 4 | ART631 elevated ROS and CHOP levels and reduced MCL1 expression in MOLM14 AML cells. (A) MOLM14 AML cells were pre-treated with ROS detection reagent CM-H2DCFDA (5 mM) for $30 \mathrm{~min}$, pre-treated with deferoxamine mesylate (DFO; $11 \mathrm{mM}$ ) for $60 \mathrm{~min}$, and then treated with ART631 (200 nM) or DMSO control for 18h. They were then analyzed by flow cytometry. Graph represents \%CM-H2DCFDA fluorescence compared to vehicle (DMSO) control treated cells. Error bars represent SD from 3 independent experiments. (B) MOLM14 AML cells were treated for $18 \mathrm{~h}$ with vehicle, AS (10 uM), ART838 (200 nM), ART631 (200 nM), cytarabine (AraC; $150 \mathrm{nM})$, or SOR (5 UM). Protein was isolated and western blotted for MCL1, BCL2, CHOP, and $\beta$-actin. (C) MOLM14 cells were treated as in (B) for 18h, total RNA isolated, CDNA synthesized, and SYBR Green GRT-PCR performed in triplicates. Ct values were normalized to housekeeping gene GAPDH and fold change shown relative to vehicle (DMSO) control. Error bars represent SD from 3 independent experiments done in triplicate.

\section{DISCUSSION}

ART838 was previously shown to have superior potency against AML in vitro and in vivo, as compared to AS, as monotherapy (17). ART838 was also shown to synergize with both VEN and SOR in vitro and in vivo $(17,20)$, most notably in xenograft models $(20)$, and to have superior pharmacologic parameters (17). However, ART838 is nearing the end of its patent life, reducing its appeal for the commercial investment necessary to develop a new drug.

The class of 2C-ARTs offered promise, as they were predicted to have similar or better pharmacologic properties compared to
ART838, based on their lower molecular weights, improved functionality, and adherence to Lipinski's rules. Indeed, multiple 2C-ART derivatives had previously demonstrated potent in vivo efficacy in a malaria model (19), suggesting good drug-like pharmacology. Their extended patent protection, physicochemical features, and in vivo antimalarial activity indicated that 2C-ART derivatives might be more attractive for development as antineoplastics, compared to earlier ART derivatives including ART838. We therefore wished to identify a new, commercially viable $2 \mathrm{C}$-ART with potential to be a key component of a novel, effective, low-toxicity AML treatment paradigm and a valuable addition to the current limited antileukemic therapeutic armamentarium. We selected two 2C-ARTs - ART576 and ART631 - for evaluation in comparison to ART838, because of their in vivo antimalarial activity and ease of synthesis.

ART631 was more active than ART576 and had similar in vitro activity as ART838 against multiple AML cell lines (Table 2). Synergism of ART838 with SOR and VEN was formally reported in vitro previously (20). Here, we set out evaluate whether ART631 adds to SOR and VEN, and our results indicate that these 3 drugs cooperate to enhance apoptotic AML cell death (Figure 3). The amount of drug cooperativity of the ART631-containing SAV 3drug combination may have been obscured in these assays by the high activity of the 2-drug SOR plus VEN and ART631 plus VEN combinations (20). ART631-containing SAV was potently active in the two tested AML cell line xenograft models, causing deep responses in MV4;11 and MOLM14 xenografts at day 7, which persisted for months during and after treatment completion and were overall comparable to responses seen in simultaneous experiments with ART838-containing SAV in xenograft models (20). Strikingly, SAV with ART631 appears to have cured 9 of 10 MV4;11 xenografted mice (Figure 7) and 2 of 10 MOLM14 xenografted mice (Figure 8). ART631-containing SAV was also similarly clinically tolerable compared with ART838-containing SAV (20). It is important to note here that we demonstrated previously that VEN plus SOR was extremely potent against MV4;11 and MOLM14 xenografts, possibly reducing the demonstrable additive or synergistic contributions of both ART631 and ART838 in their respective SAV regimens (20).

In contrast to the impressive sensitivity of these two AML cell line xenograft models to SAV regimens containing either ART631 or ART838, the AML45 PDX model, which was derived from an older adult with relapsed, refractory AML (20), was much less sensitive to all tested drug combinations. However, ART631-containing SAV was clearly the most leukemia growth inhibitory regimen and resulted in longer survival than both ART838-containing SAV (20) and the SOR plus VEN 2-drug combination (Figure 9C). These in vivo results in the AML PDX model confirm the observed in vitro cooperativity between the SAV drugs as well as the contribution of ART631 to the SAV regimen in a subtype of AML that is notoriously resistant to treatment. We propose that SAV containing ART631 (or potentially other 2C-ARTs) offers a good starting point for a future novel, low-toxicity treatment paradigm for AML that can potentially be improved upon with either additional synergistic agents or with substitutions for 


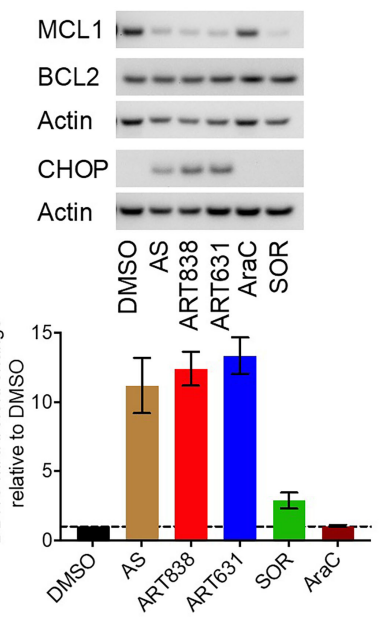

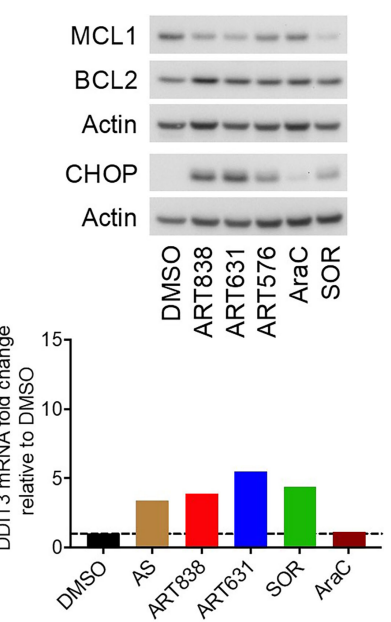

FIGURE 5 | ART631 elevated ROS and CHOP levels and reduced MCL1 expression in MV4;11 and ML2 AML cells. (A) MV4;11 cells (left) and ML2 cells (right) were treated for 18h with vehicle, AS (10 uM), ART838 (200 nM), ART631 (200 nM), cytarabine (AraC; $150 \mathrm{nM})$, or SOR (5 uM). Protein was isolated and western blotted for MCL1, BCL2, CHOP, and $\beta$-actin. (B) MV4;11 cells (left) and ML2 cells (right) were treated as in (A) for 18h, total RNA isolated, cDNA synthesized, and SYBR Green qRT-PCR performed. Ct values were normalized to housekeeping gene GAPDH and fold change shown relative to vehicle (DMSO) control. Error bars represent SD from 3 independent experiments done in triplicate. ML2 cell data is from one experiment done in triplicate.

either SOR or VEN; namely, with more potent and selective kinase and BCL2 inhibitors, respectively (26-29).

ART631 was acceptably stable in microsomes, though it lacked the robust stability of ART838 (17). ART631 exhibited similar pharmacokinetics as ART838; the multiple day MTD regimen of ART631 (15 mg/kg/d x5) resulted in similar exposure (Figure 6 and

Table 4) as that previously shown for the multiple day MTD regimen of ART838 (50 mg/kg/d x5) (20). When administered as

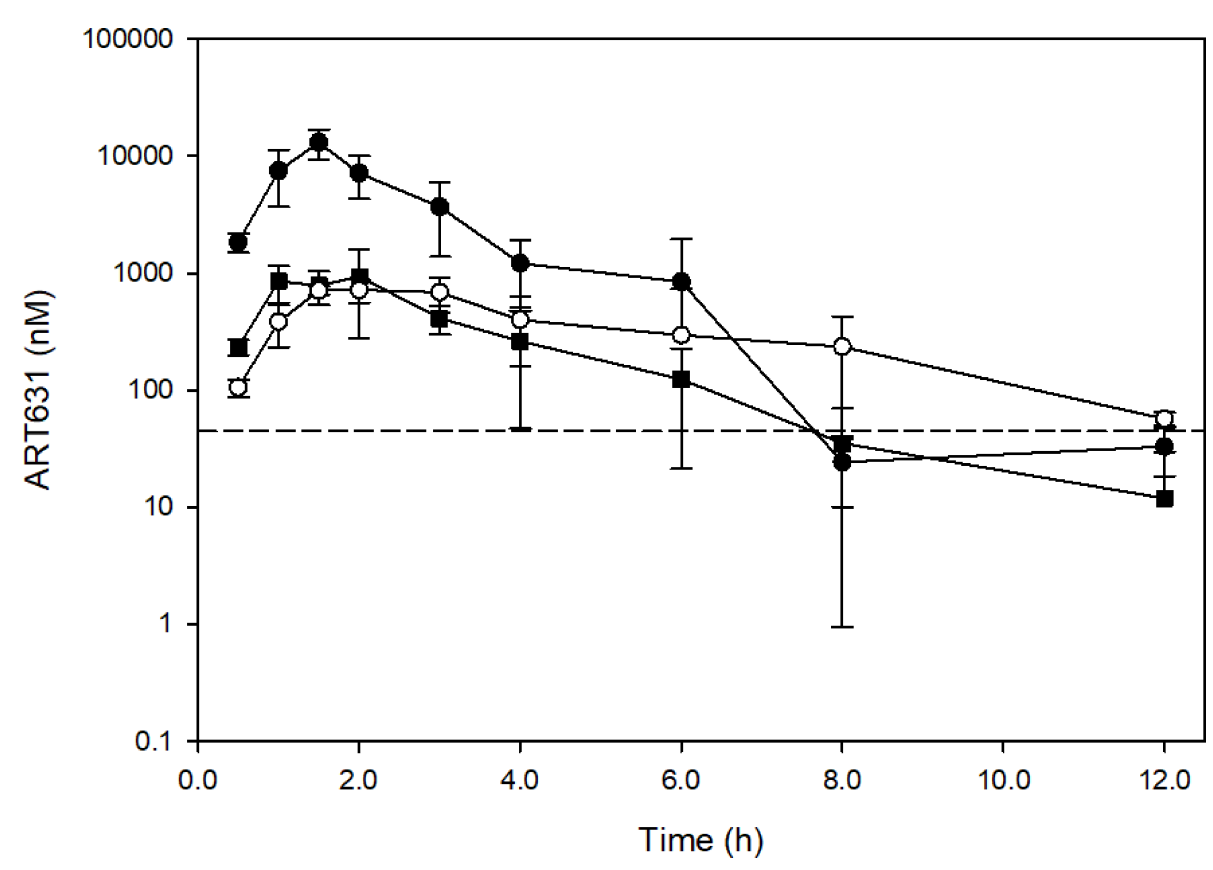

FIGURE 6 | Concentration-time profiles of ART631 in NRG mice $(n=3)$ treated with single doses of $75 \mathrm{mg} / \mathrm{kg}$ PO ART631 (MTD dose; closed circle), or multiple doses of $15 \mathrm{mg} / \mathrm{kg}$ PO ART631 for 5 days (MTD dose) alone (open circle) or in the SAV combination (closed square). Plasma was obtained over $12 \mathrm{~h}$, with ART631 concentration determined by LC/MS-MS. Dashed line represents the highest in vitro IC ${ }_{50}$ of ART631 ( $45 \mathrm{nM}$ ). Data points and error bars represent mean and SD of 3 replicates, respectively. 
TABLE 4 | PK parameters for 2C-ARTs after single dose and five-day oral regimens.

\begin{tabular}{|c|c|c|c|c|c|c|c|c|}
\hline Drug & $\begin{array}{c}\text { Dose } \\
(\mathrm{mg} / \mathrm{kg} / \mathrm{d})\end{array}$ & Schedule & $\begin{array}{l}\mathrm{C}_{\max } \\
(\mathrm{nM})\end{array}$ & $\begin{array}{c}T_{\max } \\
\text { (h) }\end{array}$ & $\begin{array}{l}\text { AUC }_{\text {last }} \\
\left(\mathrm{nM}^{\star} \mathrm{h}\right)\end{array}$ & $\begin{array}{c}T_{1 / 2} \\
\text { (h) }\end{array}$ & $\begin{array}{c}\text { Vz/F } \\
(\mathrm{L} / \mathrm{kg})\end{array}$ & $\begin{array}{c}\text { CL/F } \\
(\mathrm{L} / \mathrm{h} / \mathrm{kg}\end{array}$ \\
\hline ART631 & 75 & $\mathrm{PO} \times 1$ & 13063 & 1.5 & 22905 & 1.1 & 8.4 & 5.2 \\
\hline ART631 & 15 & $\mathrm{PO}$ qd $\times 5$ & 935 & 2.0 & 2737 & 1.7 & 21.5 & 8.7 \\
\hline ART631 in SAV & 15 & $P O$ qd $\times 5$ & 719 & 2.0 & 3734 & 2.4 & 21.3 & 6.1 \\
\hline
\end{tabular}

Pharmacokinetics were assessed in the blood plasma of NRG mice after administration of each of the noted dosing regimens (75 mg/kg ART631 PO x $1,15 \mathrm{mg} / \mathrm{kg}$ ART631 PO qd $\times 5 \mathrm{~d}$ both alone and in SAV). For reference, the maximal tolerated dose (MTD) of ART631 was determined to be $75 \mathrm{mg} / \mathrm{kg} P O \times 1 \mathrm{or} 15 \mathrm{mg} / \mathrm{kg} / \mathrm{d}$ PO $\times 5 \mathrm{~d}$. Plasma was collected at multiple time points 0.5-12 hours after the single regimens or after the $5^{\text {th }}$ dose of the five-day regimens. Samples were analyzed by liquid chromatography/triple quadrupole mass spectrometry (LC/ MS-MS) as previously described (17). PK parameters (maximum plasma concentration [ $C_{\text {max }}$; time to reach $C_{\max }\left[T_{\text {max }}\right.$; area under the plasma concentration-time curve [AUC] from time zero to the last measurable concentration [AUC last]; plasma half-life [T $1 / 2]$; apparent volume of distribution (Vz/F]; apparent clearance [CL/F]) were calculated from mean drug concentrationtime data using non-compartmental methods as analyzed in Phoenix WinNonlin version 8.3.

A

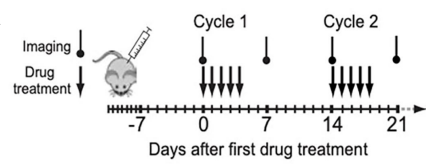

B

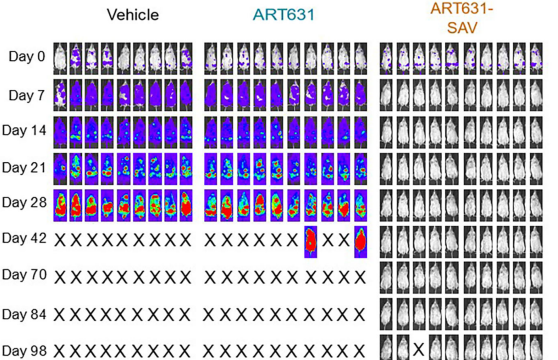

C

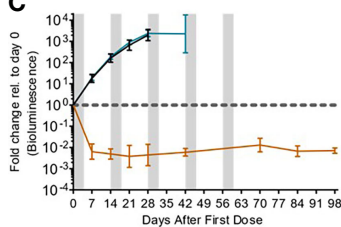

D

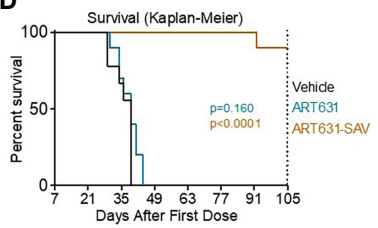

FIGURE 7 | VEN plus SOR plus ART631 induced deep, long remissions in AML xenografts. (A) SAV MTD cyclic drug treatment schema. (B) NRG mice were transplanted IV with Luc-labeled MV4;11 cells on day -10. After Xenogen quantification of baseline total body luminescence on day 0 , mice were placed into balanced experimental groups prior to drug administration via gavage, then treated per our previously established MTD PO $\times 5$ day schedule ( 5 days on, 9 days off). Mice were treated with $15 \mathrm{mg} / \mathrm{kg} / \mathrm{d}$ ART631 as monotherapy or in combination with $150 \mathrm{mg} / \mathrm{kg} / \mathrm{d}$ VEN and $30 \mathrm{mg} / \mathrm{kg} / \mathrm{d}$ SOR for 5 identical 5 -day treatment cycles over 10 weeks. These xenograft experiments with ART631 and ART631-containing SAV were performed simultaneously with those with ART838 and ART838-containing SAV reported previously (20). Treatment response outcomes were measured by (C) leukemia response quantitation (fold-change in leukemia burden on day 7 (and later) vs day 0 for each mouse via Xenogen imaging (grey-shaded bands on graphs indicate days when mice were treated). Error bars represent SD of fold-change in leukemia burden of mice in each treatment group. (D) shows corresponding Kaplan-Meier survival curves. Mouse deaths empirically defined to have occurred for reasons other than leukemia (i.e. deaths of mice with luminescence at or below average background luminescence $\left(5.7 \times 10^{5}\right.$ photons/mouse based on intensity values from non-leukemia bearing mice) were censored. Comparisons between treatment groups were calculated by log-rank (Mantel-Cox) test.

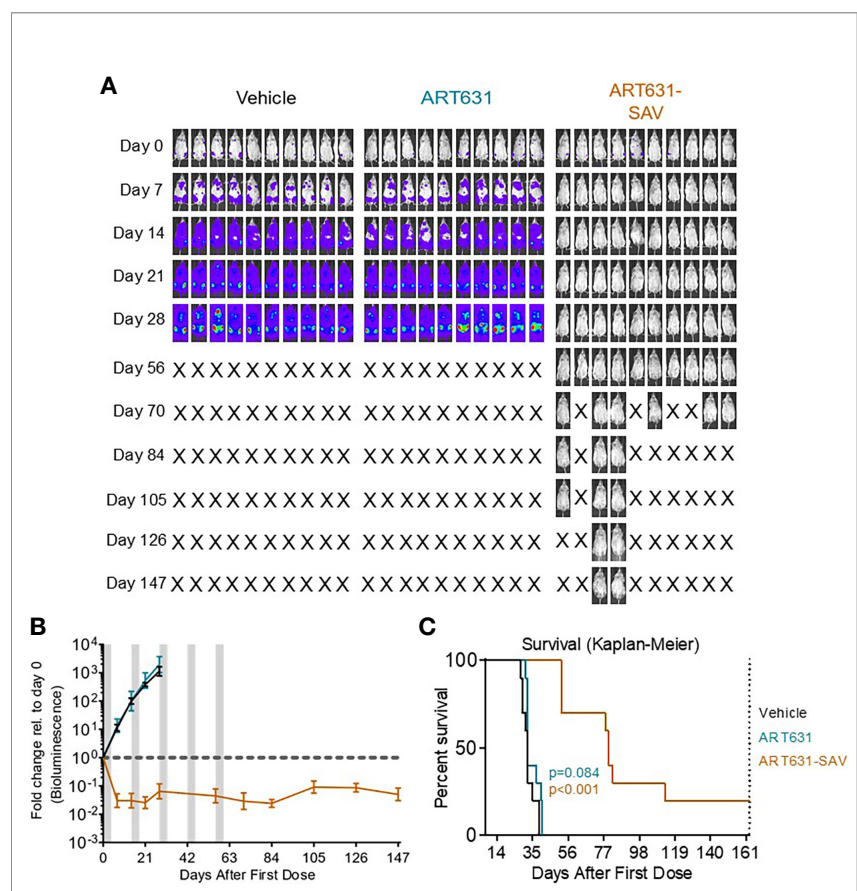

FIGURE 8 | VEN plus SOR plus ART631 induced deep, long remissions in AML xenografts. (A) NRG mice were transplanted IV with Luc-labeled MOLM14 cells on day -10. After Xenogen quantification of baseline total body luminescence on day 0 , mice were placed into balanced experimental groups prior to drug administration via gavage, then treated per our previously established MTD PO $\times 5$ day schedule ( 5 days on, 9 days off). Mice were treated with $15 \mathrm{mg} / \mathrm{kg} / \mathrm{d}$ ART631 as monotherapy or in combination with 150 $\mathrm{mg} / \mathrm{kg} / \mathrm{d}$ VEN and $30 \mathrm{mg} / \mathrm{kg} / \mathrm{d}$ SOR for 5 identical 5-day treatment cycles over 10 weeks. These xenograft experiments with ART631 and ART631containing SAV were performed simultaneously with those with ART838 and ART838-containing SAV reported previously (20). Treatment response outcomes were measured by (B) leukemia response quantitation (fold-change in leukemia burden on day 7 (and later) vs day 0 for each mouse via Xenogen imaging (grey-shaded bands on graphs indicate days when mice were treated). Error bars represent SD of fold-change in leukemia burden of mice in each treatment group. (C) shows corresponding Kaplan-Meier survival curves. Mouse deaths empirically defined to have occurred for reasons other than leukemia (i.e. deaths of mice with luminescence at or below average background luminescence $\left(5.7 \times 10^{5}\right.$ photons/mouse based on intensity values from non-leukemia bearing mice) were censored. Comparisons between treatment groups were calculated by log-rank (Mantel-Cox) test. 


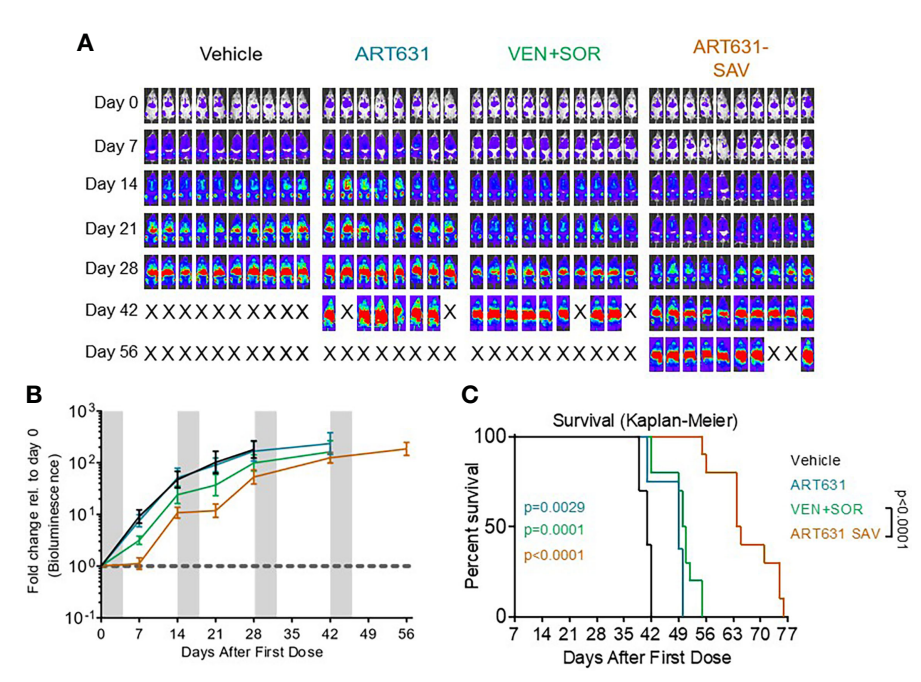

FIGURE 9 | VEN plus SOR plus ART631 inhibited growth of an AML PDX. NRG mice bearing luc/YFP-labeled AML45 PDX were prepared as in Figure 7. (A) After Xenogen quantification of baseline total body luminescence on day 0 , mice were placed into balanced experimental groups prior to drug administration via gavage, then treated per our previously established MTD PO x 5 day schedule ( 5 days on, 9 days off). Mice were treated with $15 \mathrm{mg} / \mathrm{kg} / \mathrm{d}$ ART631 as monotherapy, with $150 \mathrm{mg} / \mathrm{kg} / \mathrm{d}$ VEN and $30 \mathrm{mg} / \mathrm{kg} / \mathrm{d}$ SOR, or with ART631 plus VEN plus SOR (i.e. SAV) at the previously indicated doses for 4 identical 5-day treatment cycles over 8 weeks. This PDX experiment was also performed simultaneously with the previously reported AML45 PDX experiment with ART838 and ART838-containing SAV (20). Treatment response outcomes were measured by (B) leukemia response quantitation (fold-change in leukemia burden on day 7 (and later) versus day 0 for each mouse via Xenogen imaging (grey-shaded bands on graphs indicate days when mice were treated). Error bars represent the SD of fold-change in leukemia burden of mice in each treatment group.

(C) shows corresponding Kaplan-Meier survival curves. Comparisons between treatment groups were calculated by log-rank (Mantel-Cox) test.

part of SAV, ART631 exposure was somewhat higher and half-life longer compared with ART631 monotherapy (Figure 6 and Table 4), though fortunately increased toxicity was not observed.

While it is somewhat limited in its in vitro stability, ART631 is a first-in-class antileukemic 2C-ART with potent activity in vitro and in vivo, is easily synthesized, and is highly effective, at least serving here as a "tool" compound for proofof-principle experiments demonstrating the efficacy of a 2CART as a component of SAV. Lead optimization studies can now be performed to improve upon the efficacy, stability, and pharmacokinetic properties of ART631 or potentially other 2CARTs, in an effort to identify a lead 2C-ART. This new lead 2CART will be an essential component of a novel combination regimen that warrants clinical testing in leukemia patients.

\section{DATA AVAILABILITY STATEMENT}

The raw data supporting the conclusions of this article will be made available by the authors, without undue reservation.

\section{ETHICS STATEMENT}

The animal study was reviewed and approved by Animal Care and Use Program, University of Maryland Baltimore.

\section{AUTHOR CONTRIBUTIONS}

AK: Writing - Original Draft Preparation (equal), Writing - Review \& Editing. BSM: Methodology, Data Curation, Formal Analysis, Writing - Original Draft Preparation. (equal), Writing - Review \& Editing. BTM: Methodology, Writing - Original Draft Preparation (equal), Writing - Review \& Editing. GR: Methodology, Writing Review \& Editing. NA: Methodology, Writing - Review \& Editing. MR: Conceptualization (equal), Formal Analysis, Resources, Supervision, Writing - Original Draft (supporting), Writing Review \& Editing. CC: Conceptualization (equal), Resources, Supervision, Writing - Original Draft (supporting), Writing Review \& Editing. All authors contributed to the article and approved the submitted version.

\section{FUNDING}

This study was supported in part by the Maryland Technology Development Corporation (TEDCO) MII Innovation Grant 131175, an award from the Emmert Hobbs Foundation Endowment (BSM), and the Clinical Pharmacology Training Program grant (ABK, NIH T32GM066691). The project described was also supported by the Analytical Pharmacology Core of the Sidney Kimmel Comprehensive Cancer Center at Johns Hopkins (NIH P30CA006973 (NA, MR) and UL1TR003098 (NA, MR), Shared Instrument Grant S10RR026824 (MR). Grant Number UL1TR003098 is from the 
National Center for Advancing Translational Sciences (NCATS), a component of the NIH, and NIH Roadmap for Medical Research. Funding for the study described in this publication was provided by

\section{REFERENCES}

1. Kantarjian HM, Short NJ, Fathi AT, Marcucci G, Ravandi F, Tallman M, et al. Acute Myeloid Leukemia: Historical Perspective and Progress in Research and Therapy Over 5 Decades. Clin Lymphoma Myeloma Leuk (2021) 21 (9):580-97. doi: 10.1016/j.clml.2021.05.016

2. Sasaki K, Ravandi F, Kadia TM, DiNardo CD, Short NJ, Borthakur G, et al. De Novo Acute Myeloid Leukemia: A Population-Based Study of Outcome in the United States Based on the Surveillance, Epidemiology, and End Results (SEER) Database 1980 to 2017. Cancer (2021) 127(12):2049-61. doi: 10.1002/ cncr.33458

3. Lai C, Doucette K, Norsworthy K. Recent Drug Approvals for Acute Myeloid Leukemia. J Hematol Oncol (2019) 12(1):100. doi: 10.1186/s13045019-0774-x

4. Konopleva M, Pollyea DA, Potluri J, Chyla B, Hogdal L, Busman T, et al. Efficacy and Biological Correlates of Response in a Phase II Study of Venetoclax Monotherapy in Patients With Acute Myelogenous Leukemia. Cancer Discov (2016) 6(10):1106-17. doi: 10.1158/2159-8290.CD16-0313

5. Scholl S, Fleischmann M, Schnetzke U, Heidel FH. Molecular Mechanisms of Resistance to FLT3 Inhibitors in Acute Myeloid Leukemia: Ongoing Challenges and Future Treatments. Cells (2020) 9(11):2493. doi: 10.3390/ cells 9112493

6. McMurry H, Fletcher L, Traer E. IDH Inhibitors in AML-Promise and Pitfalls. Curr Hematol Malig Rep (2021) 16(2):207-17. doi: 10.1007/s11899021-00619-3

7. Rudrapal M, Chetia D. Endoperoxide Antimalarials: Development, Structural Diversity and Pharmacodynamic Aspects With Reference to 1,2,4-TrioxaneBased Structural Scaffold. Drug Des Devel Ther (2016) 10:3575-90. doi: 10.2147/DDDT.S118116

8. Keiser J, Utzinger J. Artemisinins and Synthetic Trioxolanes in the Treatment of Helminth Infections. Curr Opin Infect Dis (2007) 20(6):605-12. doi: 10.1097/QCO.0b013e3282f19ec4

9. Arav-Boger R, He R, Chiou CJ, Liu J, Woodard L, Rosenthal A, et al. Artemisinin-Derived Dimers Have Greatly Improved Anti-Cytomegalovirus Activity Compared to Artemisinin Monomers. PloS One (2010) 5(4):e10370. doi: 10.1371/journal.pone. 0010370

10. He R, Mott BT, Rosenthal AS, Genna DT, Posner GH, Arav-Boger R. An Artemisinin-Derived Dimer has Highly Potent Anti-Cytomegalovirus (CMV) and Anti-Cancer Activities. PloS One (2011) 6(8):e24334. doi: 10.1371/ journal.pone. 0024334

11. Efferth T, Dunstan H, Sauerbrey A, Miyachi H, Chitambar CR. The AntiMalarial Artesunate Is Also Active Against Cancer. Int J Oncol (2001) 18 (4):767-73. doi: 10.3892/ijo.18.4.767

12. Efferth T, Sauerbrey A, Olbrich A, Gebhart E, Rauch P, Weber HO, et al. Molecular Modes of Action of Artesunate in Tumor Cell Lines. Mol Pharmacol (2003) 64(2):382-94. doi: 10.1124/mol.64.2.382

13. Hooft van Huijsduijnen R, Guy RK, Chibale K, Haynes RK, Peitz I, Kelter G, et al. Anticancer Properties of Distinct Antimalarial Drug Classes. PloS One (2013) 8(12):e82962. doi: 10.1371/journal.pone.0082962

14. Choo EF, Boggs J, Zhu C, Lubach JW, Catron ND, Jenkins G, et al. The Role of Lymphatic Transport on the Systemic Bioavailability of the Bcl-2 Protein Family Inhibitors Navitoclax (ABT-263) and ABT-199. Drug Metab Dispos (2014) 42(2):207-12. doi: 10.1124/dmd.113.055053

15. Efferth T, Verdorfer I, Miyachi H, Sauerbrey A, Drexler HG, Chitambar CR, et al. Genomic Imbalances in Drug-Resistant T-Cell Acute Lymphoblastic CEM Leukemia Cell Lines. Blood Cells Mol Dis (2002) 29(1):1-13. doi: $10.1006 / \mathrm{bcmd} .2002 .0530$

16. Drenberg CD, Buaboonnam J, Orwick SJ, Hu S, Li L, Fan Y, et al. Evaluation of Artemisinins for the Treatment of Acute Myeloid Leukemia. Cancer Chemother Pharmacol (2016) 77(6):1231-43. doi: 10.1007/s00280-016-3038-2
Geminus Therapeutics LLC. The funder was not involved in the study design, collection, analysis, interpretation of data, the writing of this article or the decision to submit it for publication.

17. Fox JM, Moynihan JR, Mott BT, Mazzone JR, Anders NM, Brown PA, et al. Artemisinin-Derived Dimer ART-838 Potently Inhibited Human Acute Leukemias, Persisted In Vivo, and Synergized With Antileukemic Drugs. Oncotarget (2016) 7(6):7268-79. doi: 10.18632/oncotarget.6896

18. Budhraja A, Turnis ME, Churchman ML, Kothari A, Yang X, Xu H, et al. Modulation of Navitoclax Sensitivity by Dihydroartemisinin-Mediated MCL1 Repression in BCR-ABL(+) B-Lineage Acute Lymphoblastic Leukemia. Clin Cancer Res (2017) 23(24):7558-68. doi: 10.1158/1078-0432.CCR-17-1231

19. Mott BT, Tripathi A, Siegler MA, Moore CD, Sullivan DJ, Posner GH. Synthesis and Antimalarial Efficacy of Two-Carbon-Linked, ArtemisininDerived Trioxane Dimers in Combination With Known Antimalarial Drugs. J Med Chem (2013) 56(6):2630-41. doi: 10.1021/jm400058j

20. Moses BS, McCullough S, Fox JM, Mott BT, Bentzen SM, Kim M, et al. Antileukemic Efficacy of a Potent Artemisinin Combined With Sorafenib and Venetoclax. Blood Adv (2021) 5(3):711-24. doi: 10.1182/bloodadvances. 2020003429

21. Zimmerman EI, Turner DC, Buaboonnam J, Hu S, Orwick S, Roberts MS, et al. Crenolanib Is Active Against Models of Drug-Resistant FLT3-ITDPositive Acute Myeloid Leukemia. Blood (2013) 122(22):3607-15. doi: 10.1182/blood-2013-07-513044

22. Rudek MA, Zhao M, Smith NF, Robey RW, He P, Hallur G, et al. In Vitro and In Vivo Clinical Pharmacology of Dimethyl Benzoylphenylurea, A Novel Oral Tubulin-Interactive Agent. Clin Cancer Res (2005) 11(23):8503-11. doi: 10.1158/1078-0432.CCR-05-1037

23. Bailer AJ. Testing for the Equality of Area Under the Curves When Using Destructive Measurement Techniques. J Pharmacokinet Biopharm (1988) 16 (3):303-9. doi: 10.1007/BF01062139

24. Yuan J. Estimation of Variance for AUC in Animal Studies. J Pharm Sci (1993) 82(7):761-3. doi: 10.1002/jps.2600820718

25. Efferth T, Giaisi M, Merling A, Krammer PH, Li-Weber M. Artesunate Induces ROS-Mediated Apoptosis in Doxorubicin-Resistant $\mathrm{T}$ Leukemia Cells. PloS One (2007) 2(8):e693. doi: 10.1371/journal.pone.0000693

26. Pan R, Ruvolo VR, Wei J, Konopleva M, Reed JC, Pellecchia M, et al. Inhibition of Mcl-1 With the Pan-Bcl-2 Family Inhibitor (-)BI97D6 Overcomes ABT-737 Resistance in Acute Myeloid Leukemia. Blood (2015) 126(3):363-72. doi: 10.1182/blood-2014-10-604975

27. Khaw SL, Suryani S, Evans K, Richmond J, Robbins A, Kurmasheva RT, et al. Venetoclax Responses of Pediatric ALL Xenografts Reveal Sensitivity of MLLRearranged Leukemia. Blood (2016) 128(10):1382-95. doi: 10.1182/blood2016-03-707414

28. Nguyen B, Williams AB, Young DJ, Ma H, Li L, Levis M, et al. FLT3 Activating Mutations Display Differential Sensitivity to Multiple Tyrosine Kinase Inhibitors. Oncotarget (2017) 8(7):10931-44. doi: 10.18632/oncotarget.14539

29. Tarver TC, Hill JE, Rahmat L, Perl AE, Bahceci E, Mori K, et al. Gilteritinib Is a Clinically Active FLT3 Inhibitor With Broad Activity Against FLT3 Kinase Domain Mutations. Blood Adv (2020) 4(3):514-24. doi: 10.1182/ bloodadvances.2019000919

Author Disclaimer: Its contents are solely the responsibility of the authors and do not necessarily represent the official view of the Johns Hopkins ICTR, NCATS or NIH.

Conflict of Interest: BTM is an inventor on patent/patent applications related to the 2C-ARTs synthesis (US20150361088A1, expiration 01/22/2034). MR and CC are inventors on patent/patent applications related the Treatment of Leukemia with Artemisinin Derivatives and Combinations with Other Antineoplastic Agents (US Patent Application Number: 14/757,433/US Patent 9,918,972/ expiration 12/23/35). BTM, CC, and MR are founders of Geminus Therapeutics LLC, serve on its Board of Directors and hold equity. Under a license agreement between Geminus Therapeutics LLC and the Johns Hopkins University, Dr. Rudek and the University are entitled to royalty distributions related to technology 
described in the study discussed in this publication. This arrangement has been reviewed and approved by the Johns Hopkins University (MR) or University of Maryland (CC) in accordance with their conflict of interest policies.

The remaining authors declare that the research was conducted in the absence of any commercial or financial relationships that could be construed as a potential conflict of interest.

Publisher's Note: All claims expressed in this article are solely those of the authors and do not necessarily represent those of their affiliated organizations, or those of the publisher, the editors and the reviewers. Any product that may be evaluated in this article, or claim that may be made by its manufacturer, is not guaranteed or endorsed by the publisher.

Copyright (C) 2022 Kagan, Moses, Mott, Rai, Anders, Rudek and Civin. This is an open-access article distributed under the terms of the Creative Commons Attribution License (CC BY). The use, distribution or reproduction in other forums is permitted, provided the original author(s) and the copyright owner(s) are credited and that the original publication in this journal is cited, in accordance with accepted academic practice. No use, distribution or reproduction is permitted which does not comply with these terms. 